\title{
THE SIGNIFICANCE OF AXIAL STRESSES AND OUT-OF-PLANE RESTRAINTS ON THE DESIGN OF ROTORS N. R. SREENIVASAN
}

Propulsion Division. National Aeronautical Laboratory. Rangalore-560017. India

(Received 14 March 1977-1 and in revised form 26 September 1977)

\begin{abstract}
Summary-The importance of axial stresses in rotors and the effect of out-of-plane restraints at the hub, on the in-plane stresses are investigated by using the finite element technique, with a hexahedral isoparametric element. The stresses in a non-dimensional form are compared with the plane stress solution- The probable mode of deformation of rotors of various axial thicknesses is given. The use of the above method in the analysis of an asymmetric rotor is also demonstrated.
\end{abstract}

NOTATION

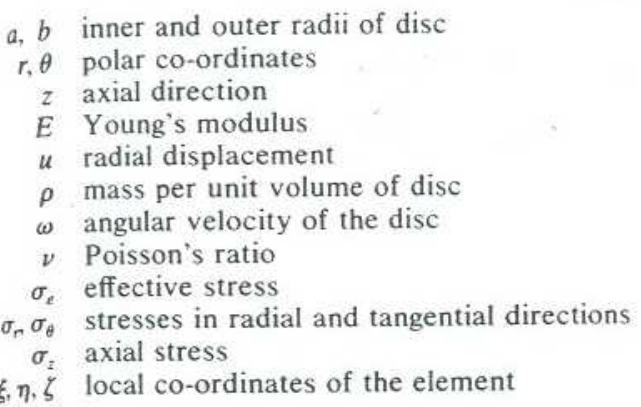

\section{INTRODUCTION}

Rotors form one of the most important structural components of a modern high speed turbomachinery power plant. They constitute a considerable portion of the total weight. The use of conventional materials in the design of these rotors has been made possible by a careful metallurgical control of the material properties. Further advances in this field can only be made by a clearer understanding of the nature and a complete picture of the stresses in the critical areas of the rotor.

Available literature on the determination of stresses in rotating discs provides exhaustive information in the case of rotors whose lateral dimensions are small and have rotational symmetry. In these conventional methods, it is assumed that the lateral thickness is small compared to the dimensions of the rotor and the presence of any axial stress and axial variation of the other stresses is neglected. Established design procedures are available for the case of thin rotors of constant and variable thickness subjected to certain simple boundary conditions and loads. However, some of the rotors encountered in actual practice are far from this type of situation. For example, integral rotors, wide hub rotors, shrunk-on rotors, asymmetric hubs and flanged discs encountered in thermal turbomachinery, have no general method of analysis. Conventional two-dimensional analysis would not be useful in predicting the critical stresses in the inner hub portion which would also be the critical section in most of the designs. These can only be determined by a three-dimensional analysis.

\section{THE IMPORTANCE OF AXIAL STRESS}

Frozen stress photoelastic experiments of Fessler, Hay and Roberts' have shown that axial stresses may be significant in discs of non-uniform thickness. They have shown that even for a disc of hyperbolic profile, the axial stresses are not negligible, although they are small. It has been reported in the same paper that axial stresses of the same order of magnitude (although of opposite sign) as the hoop stresses have been observed in the bores of gear wheels which consist of cylindrical bosses with flat plate webs. A similar situation was observed by Fessler and Thorpe ${ }^{2}$ in the case of a flanged disc. The flange caused an

†Present address: Research Engineer, Franklin Institute Research Labs., Philadelphia, PA 19103, U.S.A. 
increase of $70 \%$ in the compressive axial stress at the bore. This also changed the variation of the in-plane stresses. Reinforcement at the bore of a rotating disc by a thickened hub or a thick tapering section introduces a three-dimensional state of stress. The effectiveness of such reinforcements has been studied experimentally by Fessler and Thorpe. ${ }^{2}$ Normal calculations which ignore these axial stresses may seriously underestimate the true value of critical stresses, as these stresses occur at a location where the hoop
stresses are maximum.

Constraining the disc at the hub by attaching it to a flange fixed at one end, or shrunk-on wheels fitted on a shaft, also introduce a three-dimensional state of stress. This would also be the case for a wheel which is machined integral with the shaft. In this case, some assumptions have to be made regarding the length of the hub or shaft on either side of the wheel which is effective in reinforcing the disc, if it has to be analysed by the two-dimensional procedure. As this is made empirically, a certain degree of uncertainty would be involved in the final results.

Haigh and Murdoch ${ }^{3}$ have carried out an elastic analysis of an axially symmetric turbine wheel of appreciable thickness. This has been carried out by the numerical solution of the three-dimensional stress-strain equations using two stress functions and relaxing the governing equations. Kobayashi and Trumpler ${ }^{4}$ have solved the problem of an elastic analysis of rotors of general thickness variation. Here, the effect of temperature and any restraint on the boundary are neglected. Evaluation of the stresses and displacements in a thin uniform disc with clamped bore is carried out by Anantharamu and Janardhana Iyengar. ${ }^{5}$ The effect of out-of-plane restraint on the in-plane stress solution is accounted for by superposing an axial stress distribution which decays exponentially from the bore on the plane stress solution.

From the review of the work done, it is clearly seen that no one particular method would be suitable to analyse practical rotors encountered by a designer. As varied geometric configuration with different boundary constraints are encountered in the design stage, a general method of three-dimensional analysis would be a useful tool. Also, considerations such as non-symmetry, thermal loading, inelastic analysis and peculiar boundary conditions preclude the use of the above methods of analysis in the case of actual rotors. In the above circumstances, the most efficient and versatile method of analysis is the finite element technique.

\section{COMPUTATIONAL PROCEDURE}

An isoparametric hexahedral element has been used in the present investigations to carry out a three-dimensional analysis of a rotor subjected to the conditions and restraints mentioned in the previous section. The hexahedral element-used is of the Serendipity type as shown in Fig. 1 with its global and local coordinate axis.

The details of the shape functions of such elements as well as the analysis procedure using suct elements in the finite element technique has been well documented and will not be discussed here. Ont could refer to the works of Zienkiewicz ${ }^{6}$ for the details. To obtain a higher order element than the brick element shown in Fig. 1, nodeless variables have been introduced in addition to the usual shape functions Thermal strains are accounted for by treating them as initial strains. Body forces for the elements art evaluated using the shape functions. Provision is made in the program to account for prescribed forces anc displacements at the nodes. Problems with sliding boundaries as shown in Fig. 2 can be solved. This featurt will minimise the amount of work involved in the case of axisymmetric structures such as rotors.

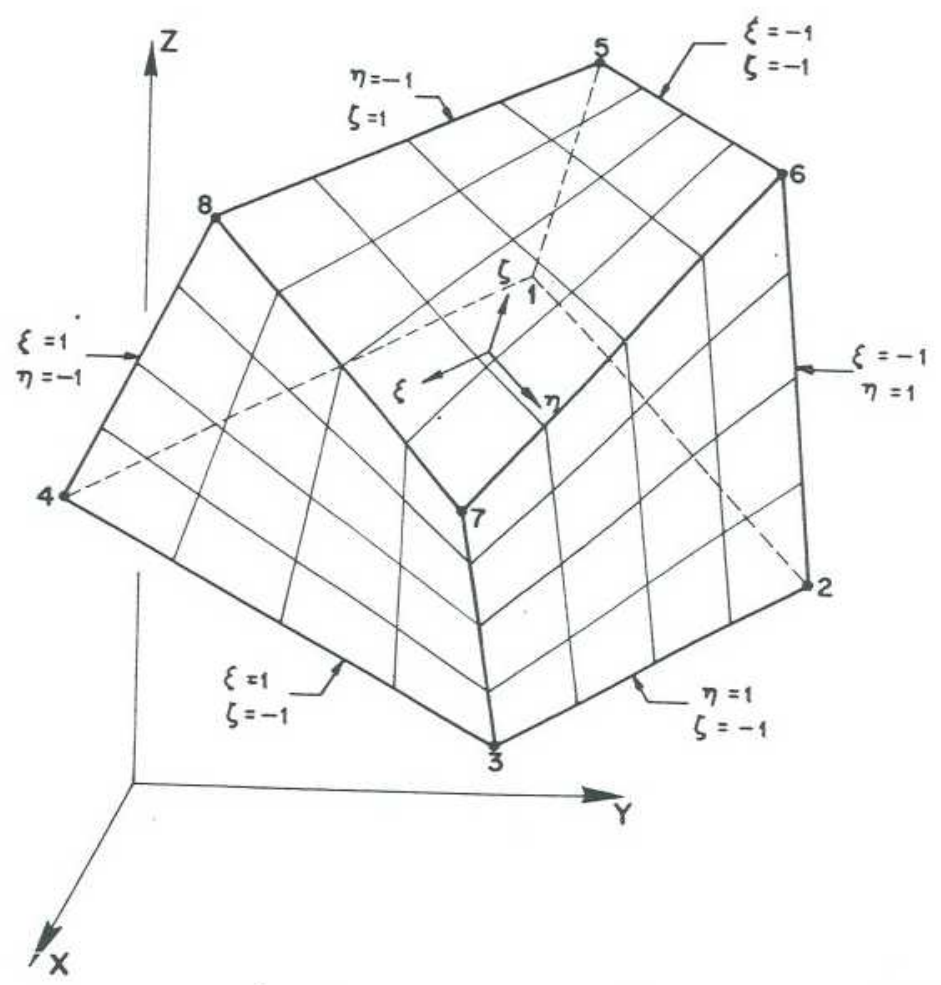

FIr. 1. Parametric and rectangular coordinate systems-eight nodal hexahedral element. 


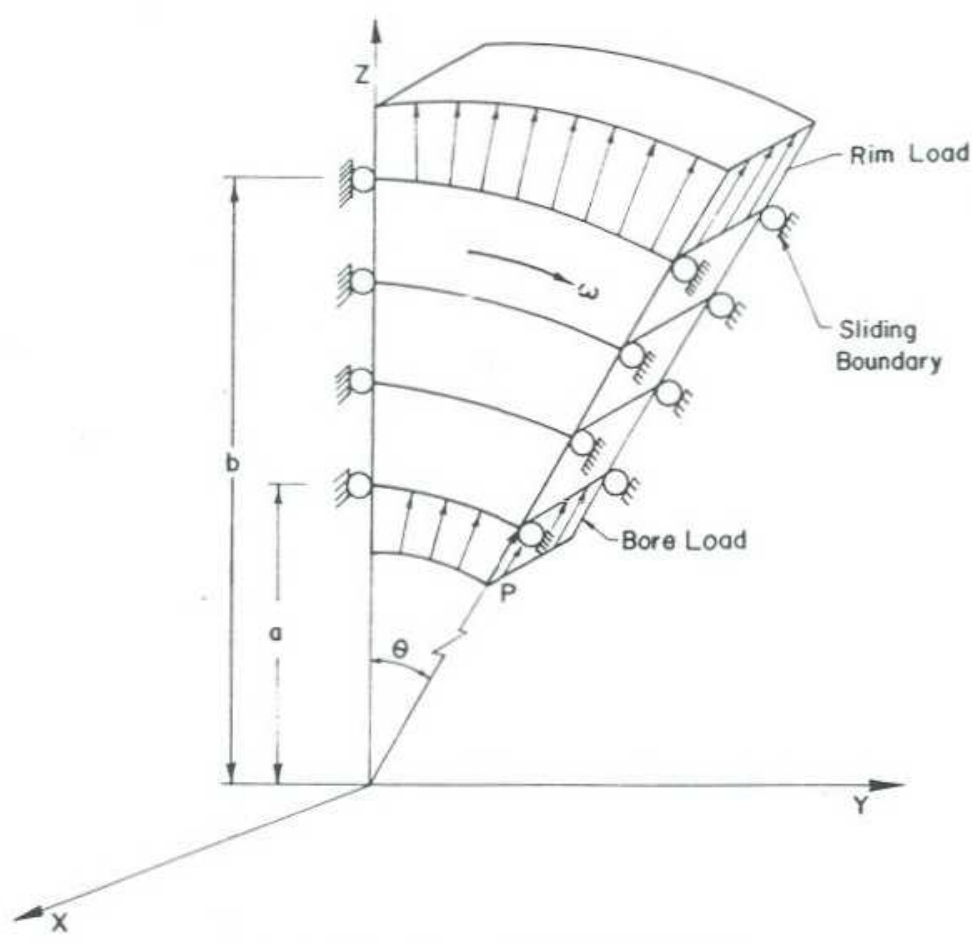

FIG. 2. Loading of an axisymmetric body.

The validity of the results from the program is checked by comparing the results of the analysis by Timoshenko ${ }^{7}$ for the case of a rotating parallel sided disc. Also, the symmetrical turbine wheel analysed by Haigh and Murdoch ${ }^{3}$ is solved by the technique developed in the present investigation. In this problem the main load on the wheel is only the radial pull of the blades, as shrinkage stresses are assumed to be zero at the running speed. The results are presented in Fig. 3. As the complete data for the wheel material such as elastic constants were not available in the work of the above authors, it was not possible to directly compare their values with the evaluated results. However, a comparison of the results obtained from the analysis with plane stress solution shows that the three-dimensional calculation gives a higher value of effective stress by about $10 \%$ as observed by Haigh and Murdoch ${ }^{3}$ in their approach. The variation of the other stresses are on similar lines to the results of the above authors. This proves the adequacy of the model representation of the rotors for the three-dimensional analysis.

\section{EVALUATION OF THE RESULTS}

The analysis of an uniform thickness annular disc is carried out for various boundary conditions. The geometry of the problem is shown in Fig. 4(a). The boundary conditions considered in the present investigation as applied to a thin disc, with the element discretization and boundary restraint condition applied to various cases considered are shown in Fig. $4(\mathrm{~b})$. The significance of the thickness, on the magnitude and the decay of the axial stresses is studied for various boundary conditions. For the sake of simplicity of the analysis and comparison, a disc of uniform thickness is chosen for the study. This would clearly demonstrate the importance of the axial thickness as well as of the out-of-plane restraints on the stresses. Further computations may be carried out for a parametric study of variable thickness rotors.

The variation of axial stress along the radius at mid-plane for various $t / a$ radios is shown in Fig. 5 . The study is carried out for the same inner to outer radius ratio of two, with the thickness in the $z$-plane increasing from $0 \cdot 1$ to $1 \cdot 0$. For the free boundary case considered here, the axial stress induced at the inner boundary is very small for a small axial thickness as expected. For a ratio of $t / a=0 \cdot 5$, an axial stress of about $5 \%$ (of the tangential stress) is observed, which increases to $34 \%$ for a $t / a$ ratio of 1.0 . The decay of the stress for the $t / a$ ratio of 1.0 is quite slow and considerable amount of axial stress will be present even beyond the usual radius at which it had become negligible for the case of a thin lamina.

Fig. 6 gives the variation of axial stress along the radius for discs of various $t / a$ ratios, where the inner radius is clamped both radially and axially. For a $t / a$ ratio of $0 \cdot 1$, axial stress which is of the same order of magnitude as tangential stress is induced, which decays very fast and becomes zero along the radius from the inner boundary. For the case of $t / a$ of 0.5 the rate of decay is slower, though the relative magnitude of the axial stress induced is the same as the previous case. For a variable thickness case plotted, the rate of decay is much slower, clearly demonstrating the importance of axial stresses in such rotors.

In the case of shrunk fit and integral rotors, there would be a certain amount of axial restraint imposed by the shaft on the inner radius. As this would also be the area of fatigue failure, an understanding of the nature of the stresses prevalent would be of considerable importance. The variation of axial stresses along the radius from the inner periphery for the condition that the bore is constrained axially and is free radially, is given in Fig. 7. This boundary condition induces an axial stress of about $20 \%$ for the $t / a$ ratios considered. The decay rates in these cases are dependant on the $t / a$ ratio under consideration.

Fig. 8 shows the variation of axial stresses at bore across the thickness of a lamina, for various axial thickness ratios. The axial thickness is varied from 0.1 to 1.5 keeping the other data same. The axial stress 


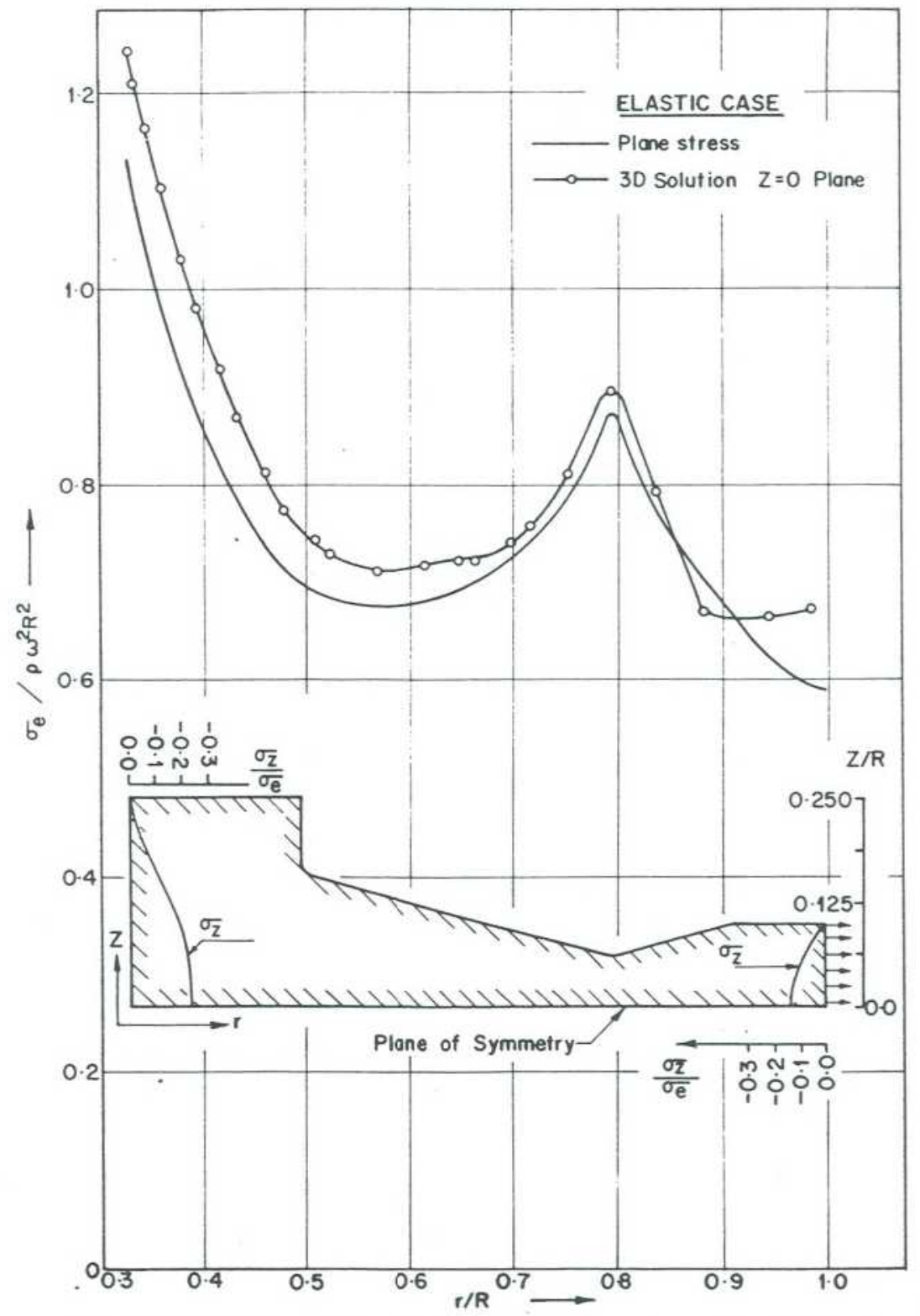

FIG. 3. Distribution of dimensionless stresses in a symmetric wheel. 

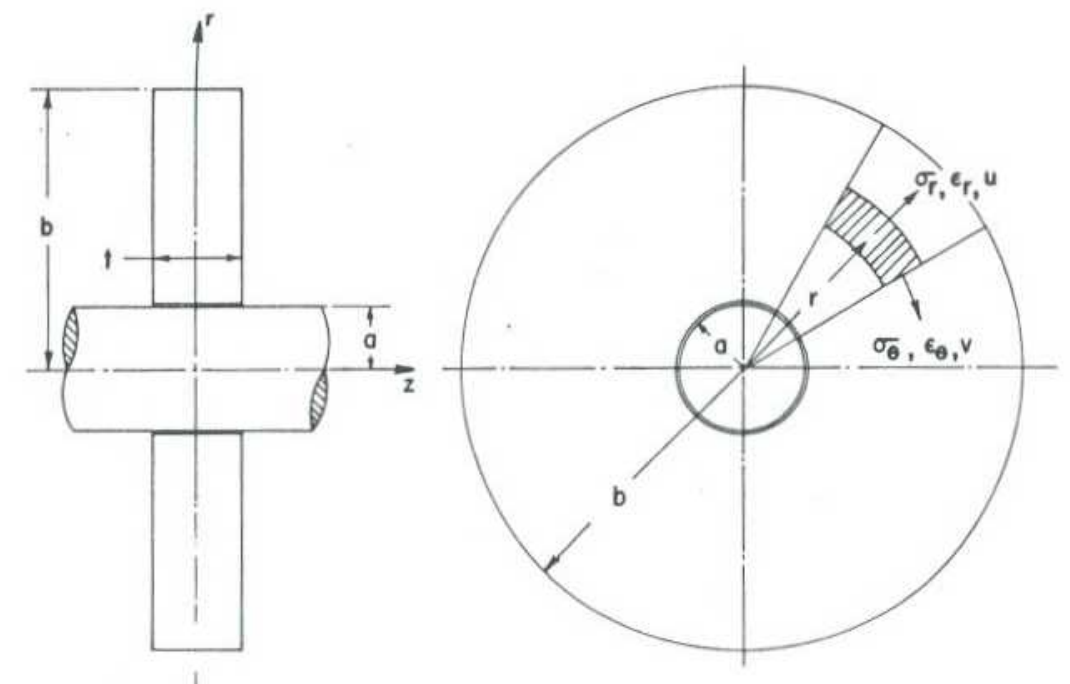

FIG. 4 (a)

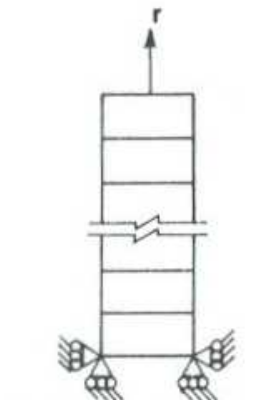

a) Free Bore

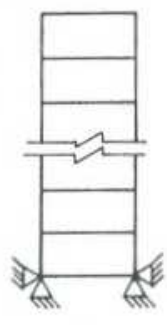

b) Clamped Bore

$$
\text { FIG. } 4 \text { (b) }
$$

Fig. 4. (a) Geometry and coordinates, (b) Boundary conditions considered.

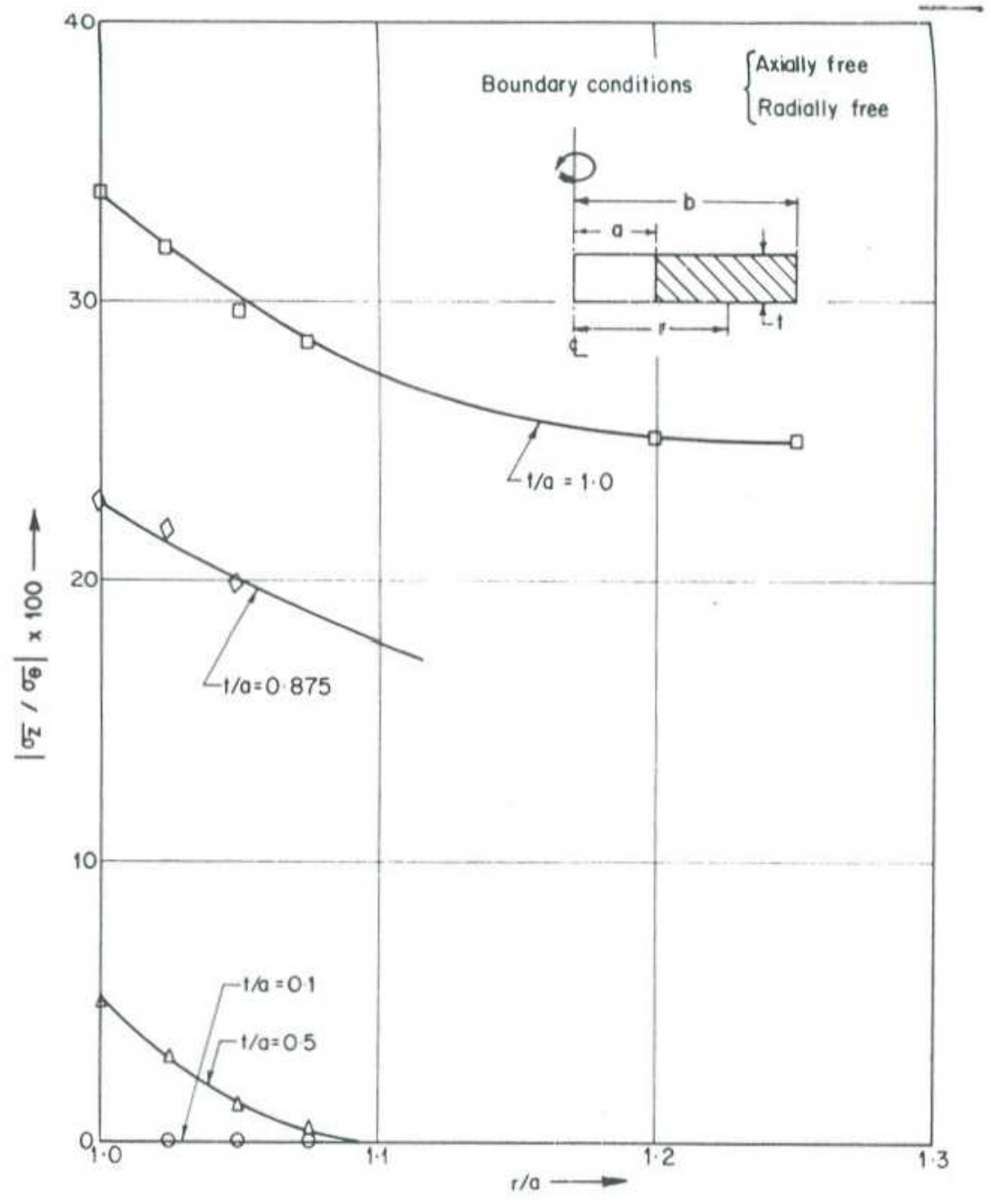

Fig. 5. Variation of axial stress along the radius at mid-plane for various $t / a$ ratios. Boundary conditions: Axially free and radially free. 


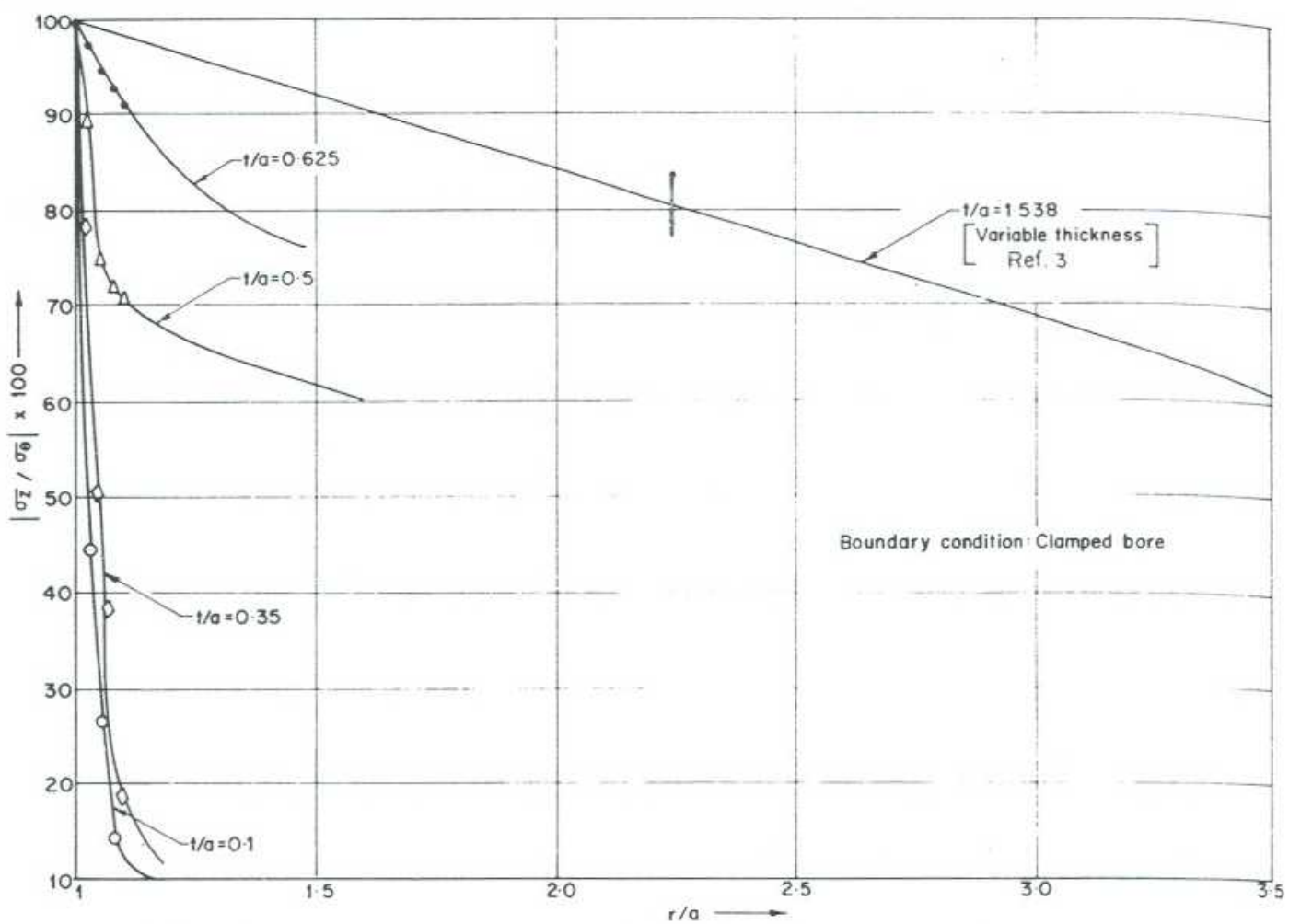

FIG. 6. Variation of axial stress along the radius at mid-plane for various $t / a$ ratios. Boundary conditions: Axially and radially clamped.

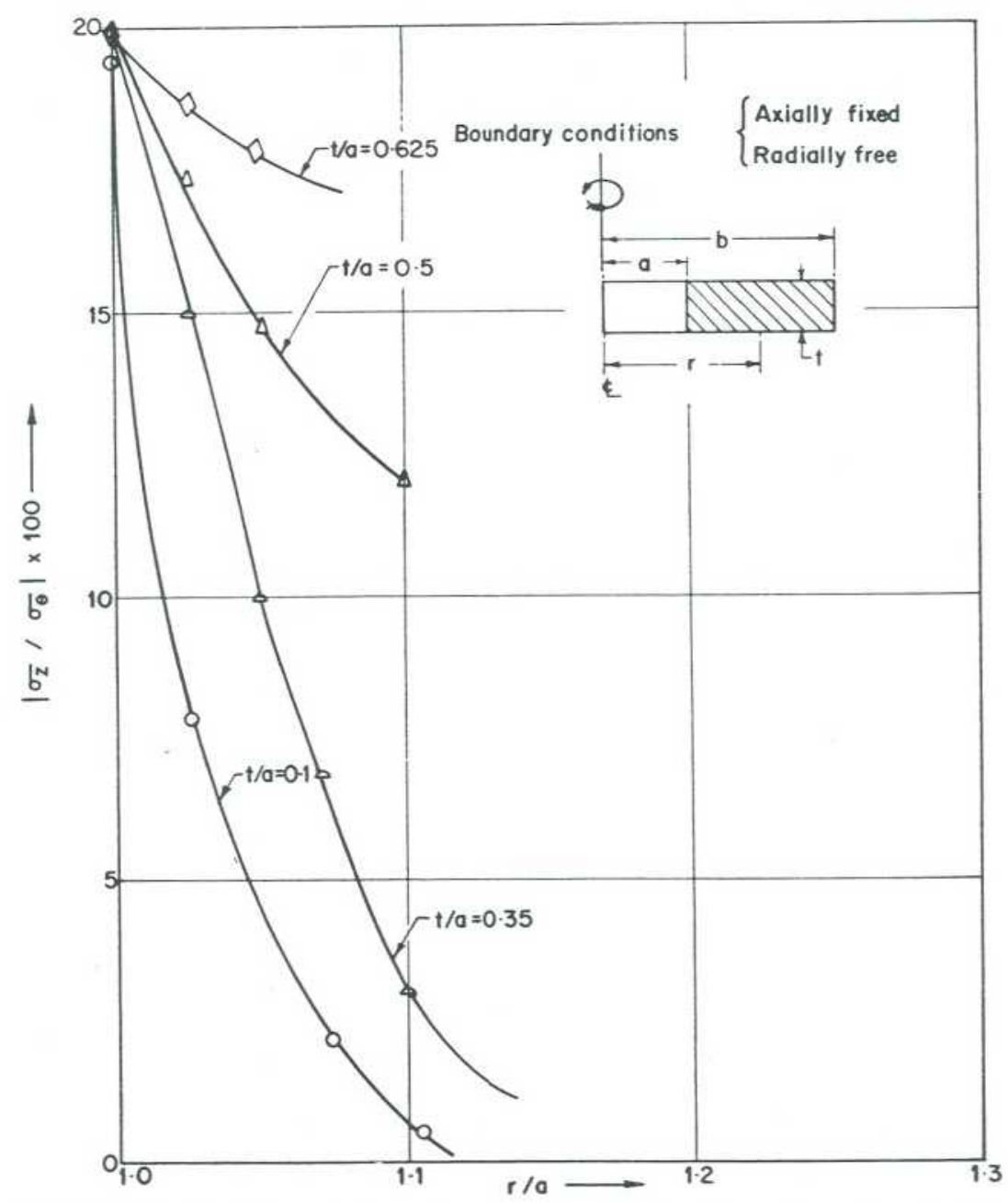

FIG. 7. Variation of axial stress along the radius at mid-plane for various $t / a$ ratios. Boundary conditions: Axially fixed and radially free. 


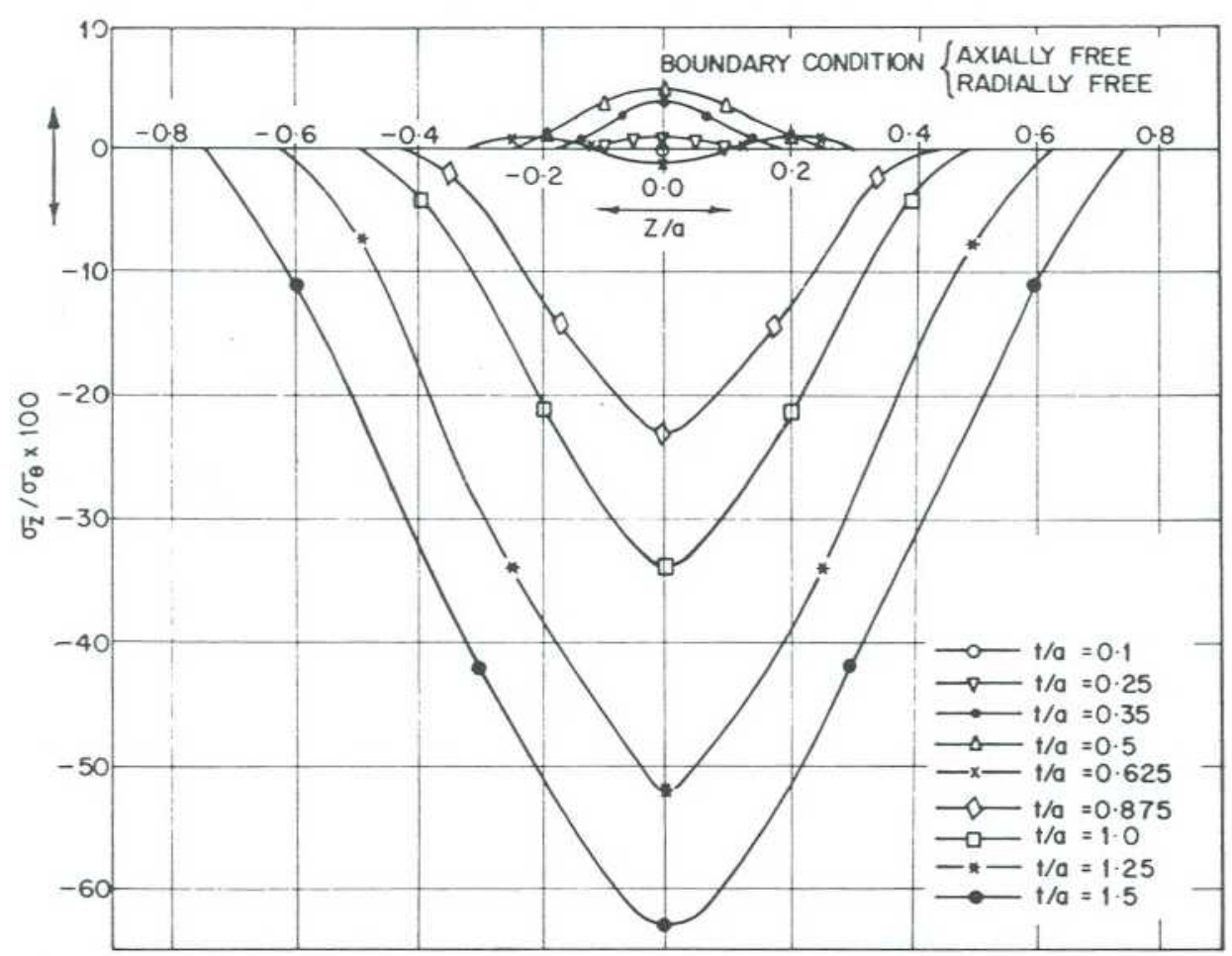

FIG. 8. Variation of axial stress across the thickness at bore. Boundary conditions: Axially free and radially free.

is maximum at the central plane in all the cases diminishing to zero on either side. For the free boundary condition case considered here, the maximum value of axial stress increases as the $t / a$ ratio increases. For example, a maximum value of $34 \%$ is observed for a thickness ratio of unity. For the axially clamped radially free case shown in Fig. 9, an approximately uniform value of $20 \%$ axial stress (w.r.t. $\sigma_{\theta}$ ) is observed for all the $t / a$ ratios considered. For the completely clamped condition a value of axial stress of the same order of magnitude as tangential stress is induced for all the ratios considered, as seen in Fig. 11. For the radially clamped and axially free case, axial stresses become more and more important as the $t / a$ ratio increases as shown in the Fig. 10. Ideal clamping in the axial or radial direction may be very difficult to achieve physically. A theoretical clamping would imply a tensile force applied in that direction. However. this condition would be able to give a bound on the stresses induced in such a case.

During the study of axial stresses, it was observed that a small and insignificant value of axial stress was present for a $t / a$ ratio of $0 \cdot 1$. The value was negative which can be explained by the contraction due to Poisson's ratio effect. However, a positive value of axial stress was observed for a $t / a$ ratio of 0.5 . A detailed investigation was carried out and the variation in magnitude and sign of the maximum axial stress at bore with respect to the various $t / a$ ratios considered is shown in Fig. 12. It is seen that for very small ratios of $t / a$, the axial stresses are negative, changing sign to positive for $t / a$ ratios between $0 \cdot 2$ and $0 \cdot 6$. For higher ratios the stresses are again negative. This phenomenon can be explained by a close look at the deformation pattern. A deformation pattern assumed in Fig. 13 explains the variation of the axial stress. In the case of medium sized hubs, it can be assumed that the disc is comprised of thin parallel laminae which deform during rotation as shown in the Fig. 13. This results in a lack of fit of the slices which necessitates a positive axial stress for compatability. However, in the case of a wide hub, as in the case of $t / a$ ratio of 1.0 , considerable axial stresses and strains are observed at the inner radii which causes the slices to take up positions as shown. This lack of equillibrium of the slices requires a negative axial stress for the lamina to be in equilibrium. This mechanism explains the peculiarity observed for the various thickness ratios considered in the investigation.

In order to demonstrate the usefulness of the technique, the nature of the stresses in an actual turbine wheel was evaluated. The numerical example presented here refers to the first high pressure stage of a $6000 \mathrm{HP}$ steam turbine asymmetric rotor designed by conventional methods, to operate at $3000 \mathrm{rev} / \mathrm{min}$. Details of the geometry, loads, temperature and elastic constants are shown in Fig. 14. Areas of importance from the point of view of axial stresses are the rim and bore. The effect of axial stresses on the in-plane stresses is shown in Fig. 15 where the variation of the effective stress along the radius at various axial planes is plotted. A plane stress solution for the same loads and geometric conditions is also shown in the same figure for comparison. It will be clearly seen that the plane stress technique is not conservative for asymmetric rotors such as the one considered here. This necessitates an analysis of the above three dimensional type.

\section{CONCLUDING REMARKS}

In the foregoing analysis, with the use of the isoparametric hexahedral element and the finite element technique, the importance of the axial stresses and the 


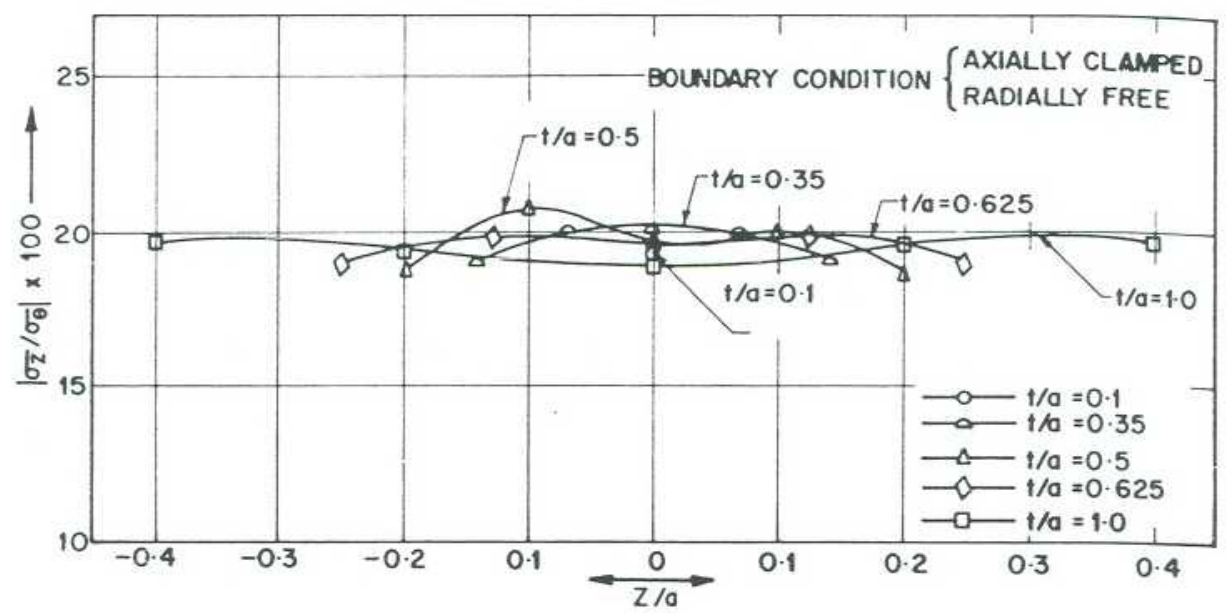

FIG. 9. Variation of axial stress across the thickness at bore. Boundary conditions: Axially clamped and radially free.

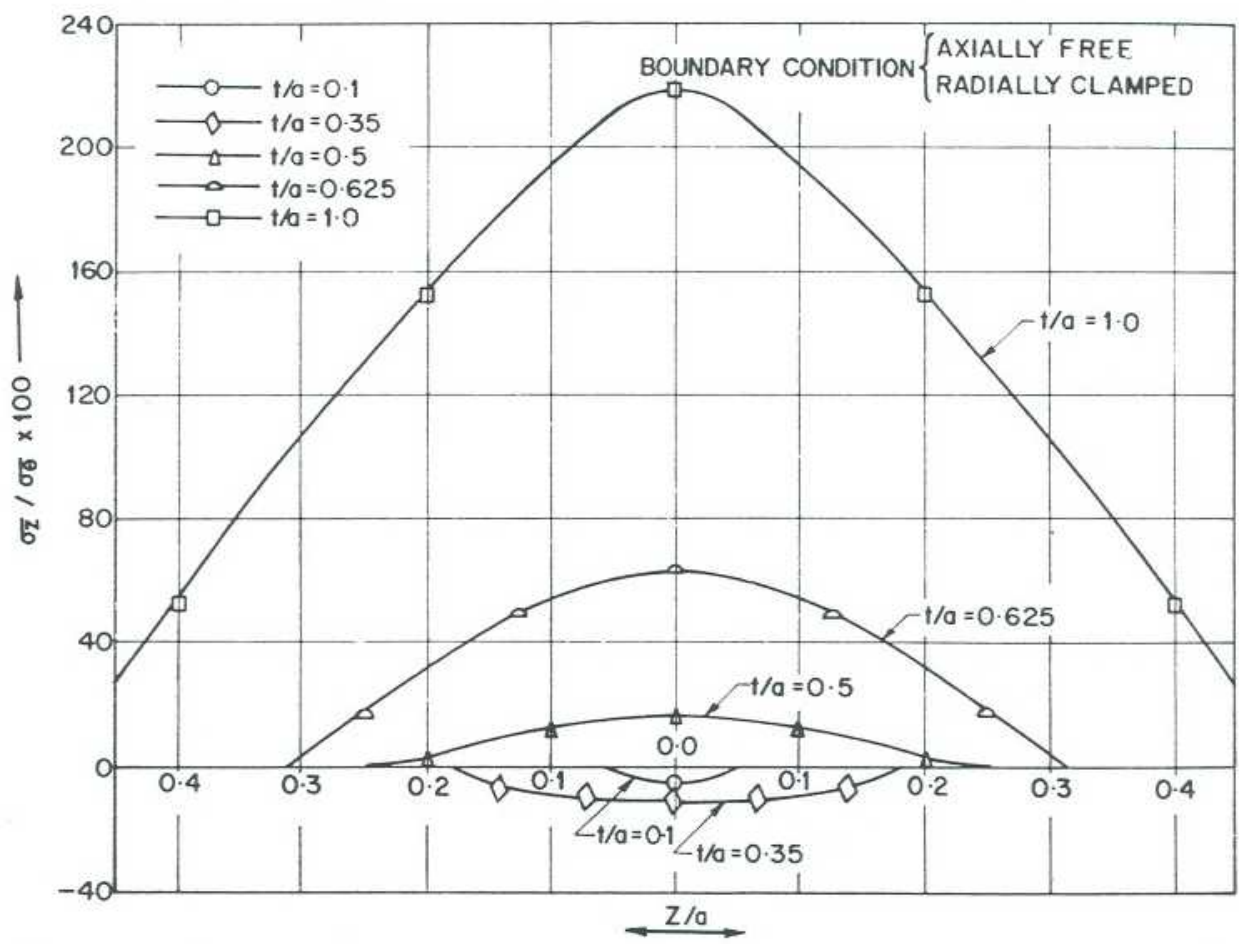

FIG. 10. Variation of axial stress across the thickness at bore. Boundary conditions: Axially free and radially clamped.

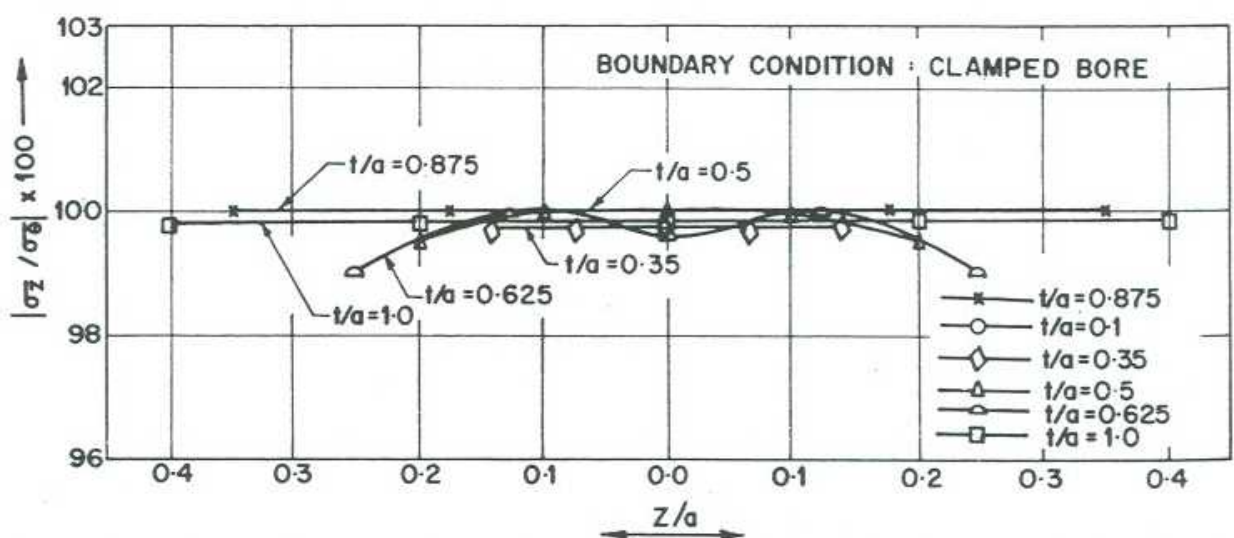

FIG. 11. Variation of axial stress across the thickness at bore. Boundary conditions: Axially and radially clamped. 


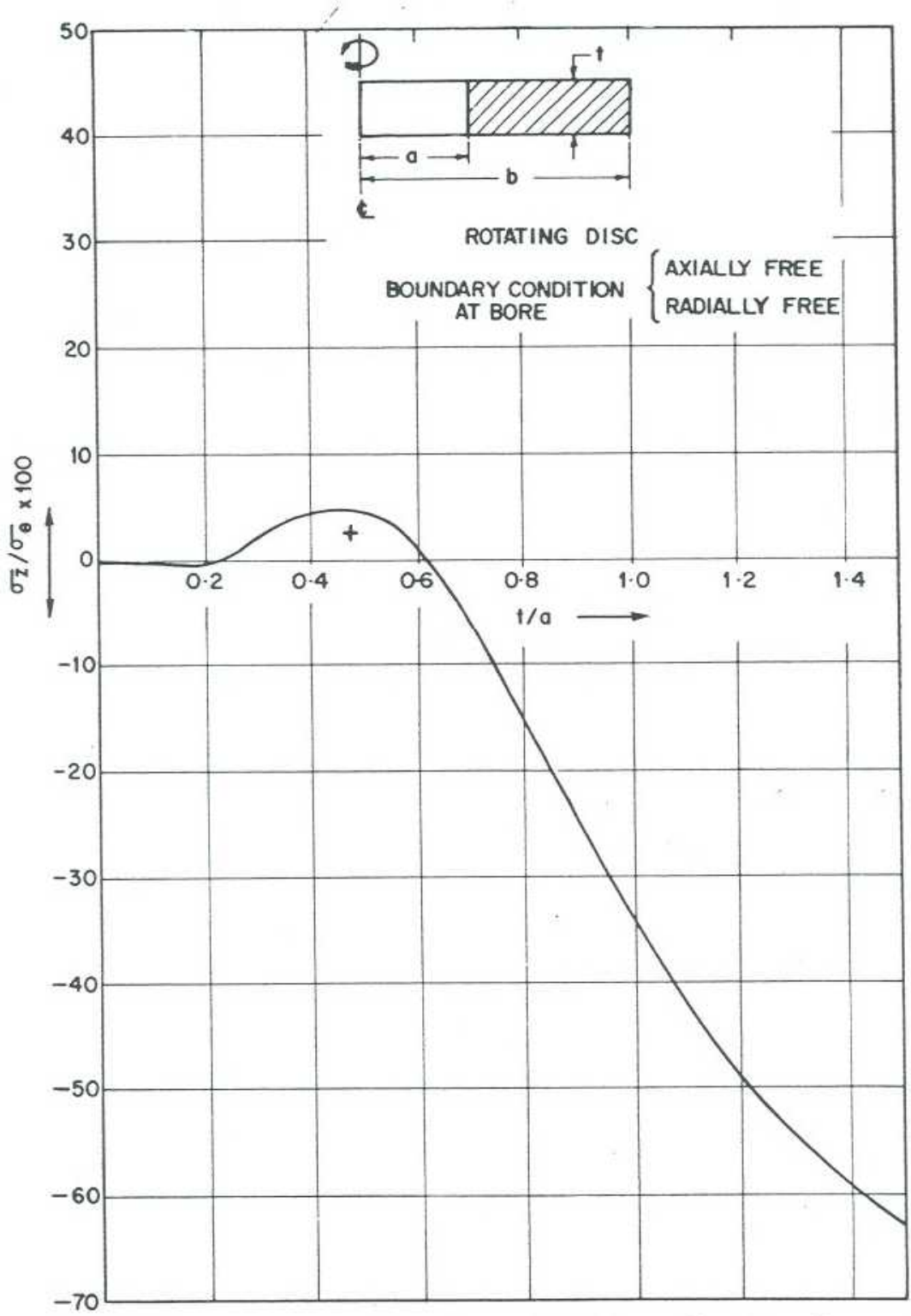

FIG. 12. Maximum axial stress at bore for various axial thickness discs. Boundary conditions: Axially and radially free. 

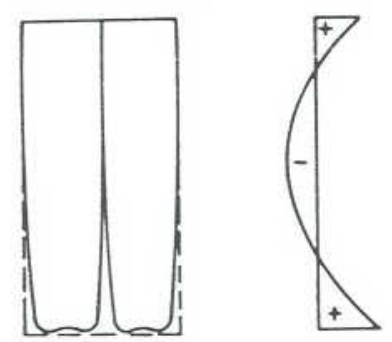

Lock of fit of slices
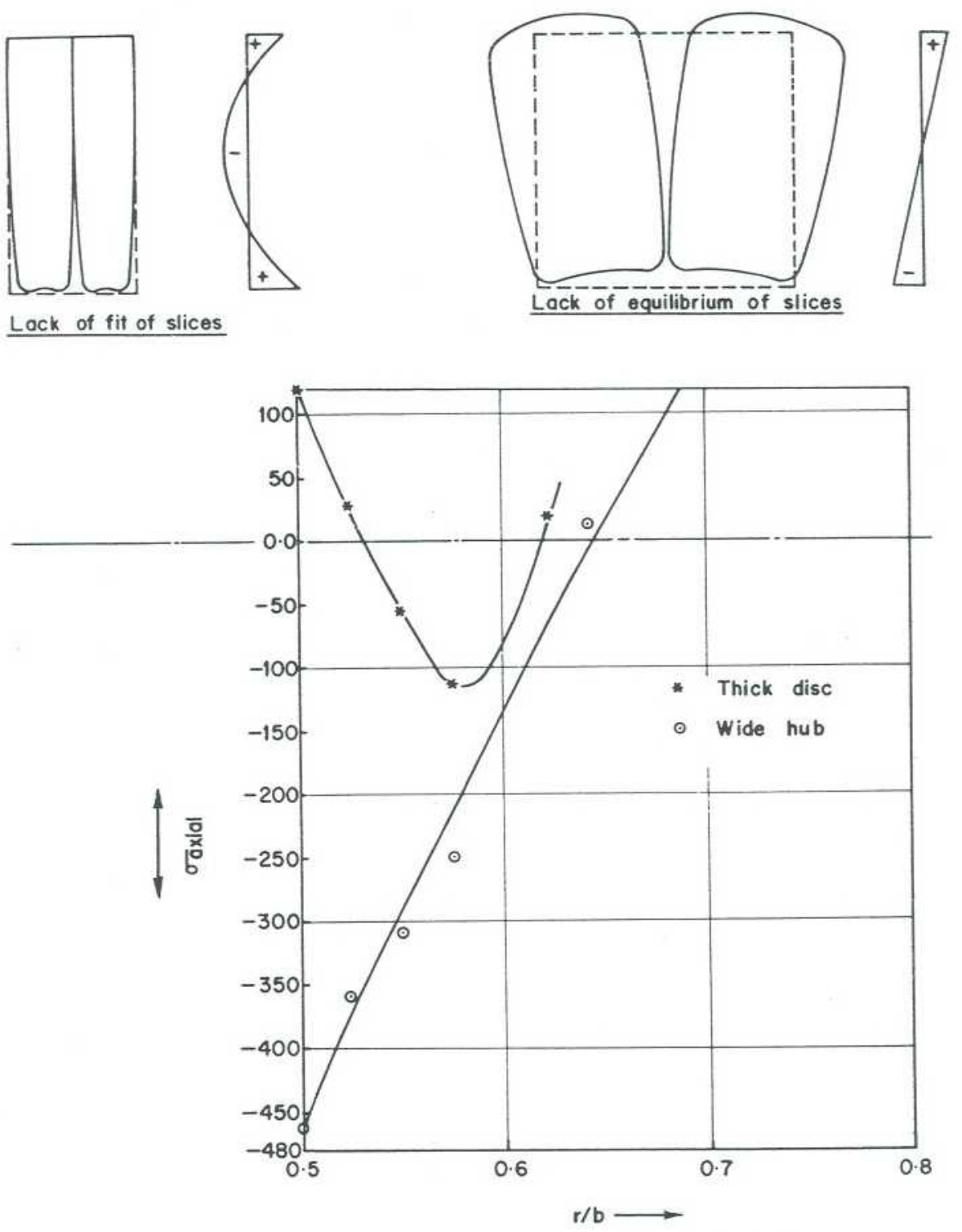

FIG. 13. Mechanism of deformation of parallel sided discs. 


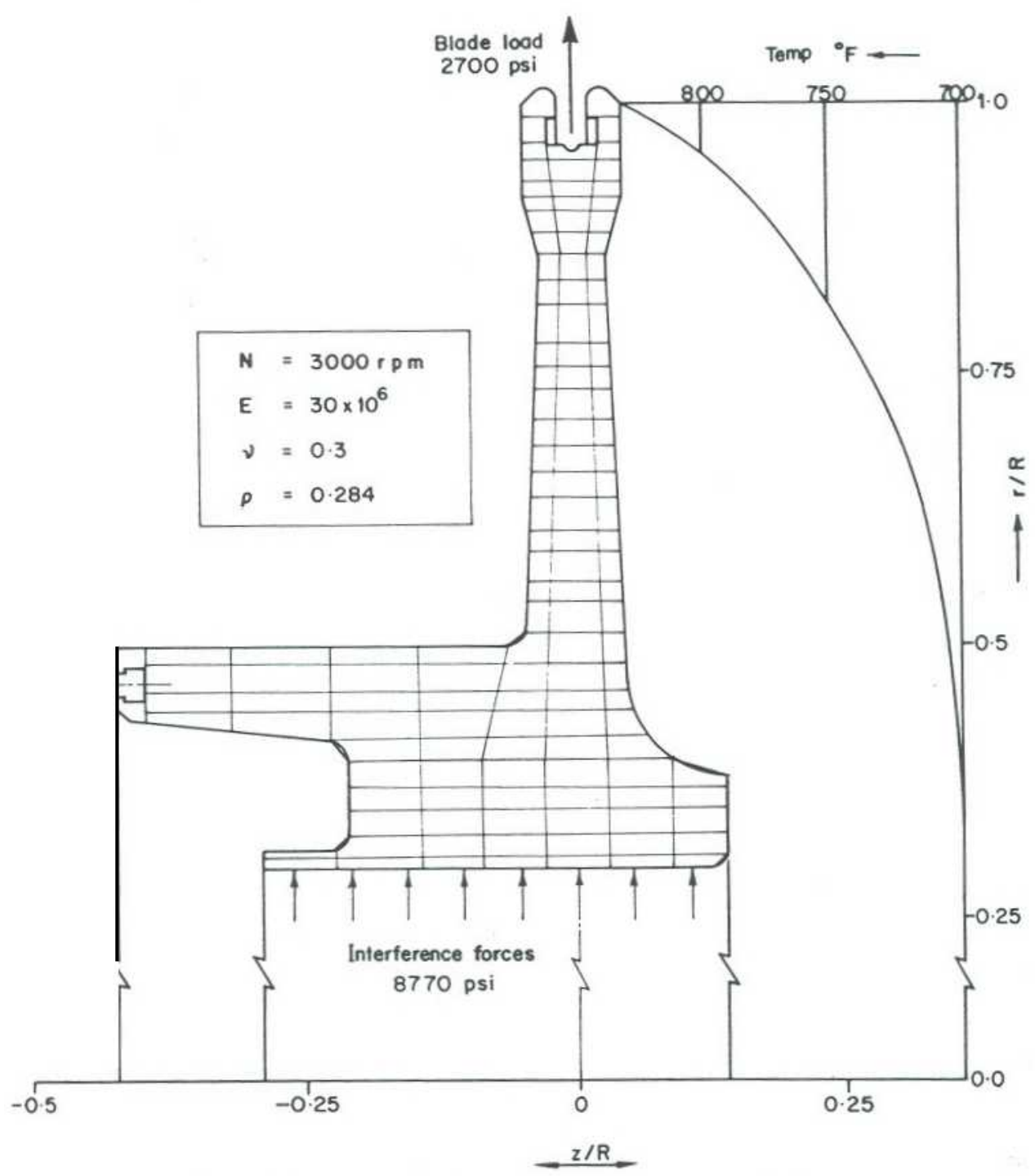

Fig. 14: Typical high pressure asymmetric turbine wheel. 


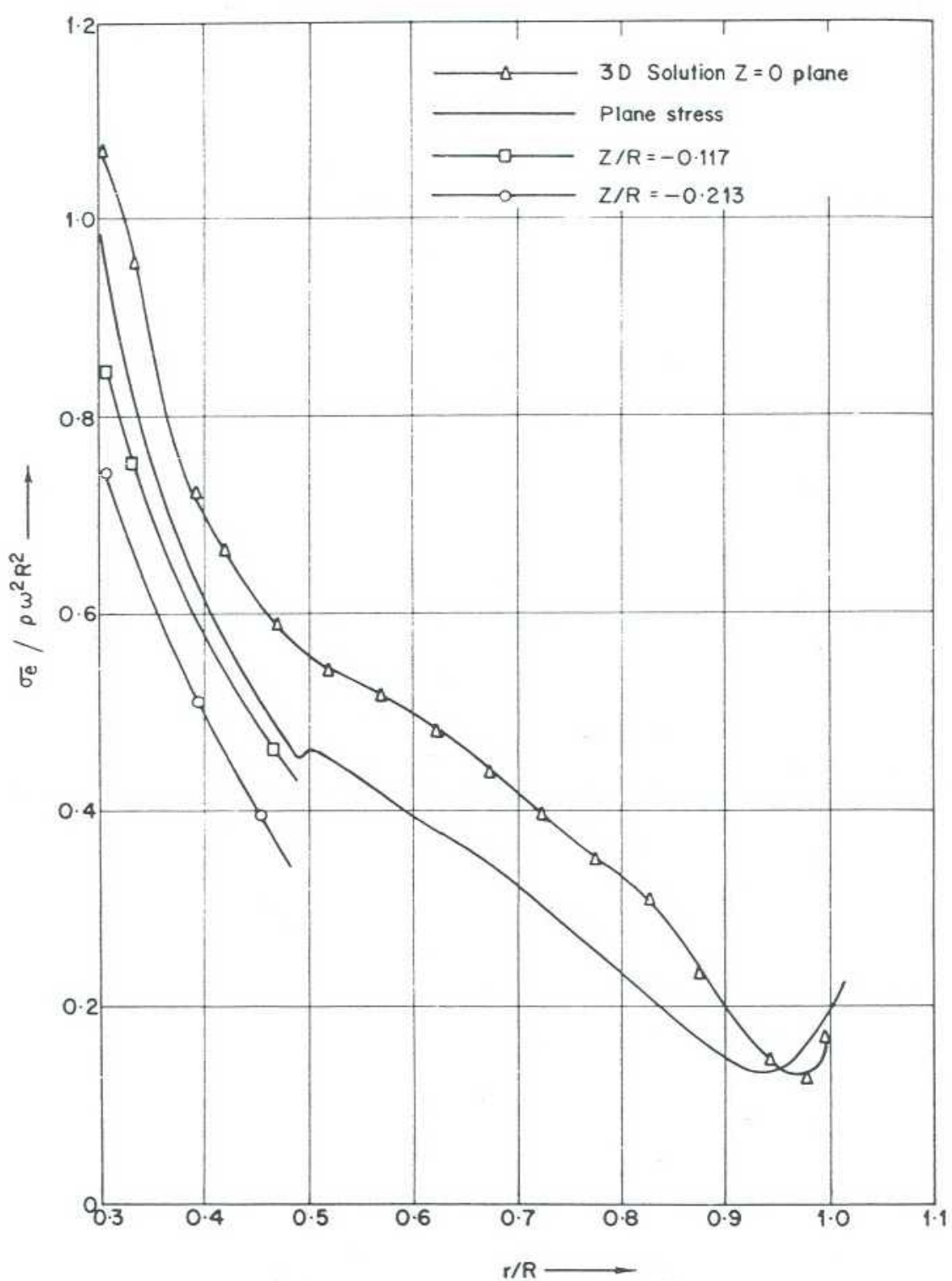

Fia. 15. Radial distribution of effective stress on several axial planes of an asymmetric turbine wheel,

out-of-plane restraints on the three-dimensional stresses of uniform thickness rotors as well as asymmetric rotors is elucidated. It has been shown that the axial stresses become significant even in the case of uniform thickness discs when the axial dimension becomes appreciable. Also, out-of-plane restraints have been shown to he of importance in the analysis of rotors and such conditions may effect and even change the nature and pattern of the in-plane stresses.

\section{REFERENCES}

I- H. FESSLER, E. 1. HAY and E. A. ROBERTS, Centrifugal stresses at the bores of wheels. Engineer 211. 113 (1961).

2. hl. FESSLER, and T. E. THORPE, Centrifugal stresses in rotationally symmetric gas turbine discs. J. Strain Anal. 3(2) (1968)-

3, R. E. HAEGH and M. L. MURDOCH, Analysis of centrifugal stresses in turbine wheels. J. Mech. Engng Sci. 5, 66 (1963).

4. A. S. KOBAYASHI and $P_{-} R_{-}$TRUMPLER. Elastic stresses in a rotating disc of general profile. Int. J. Aleck Sci. 2, 13-29 (1960).

5. S. ANANTHAPAMU and K. JANARDHANA IYENGAR, Quasi-three-dimensional elastic stresses in rotating disks. Int. J_Mech. Sci_16, 473-477 (1974).

6. 0. C. Z1ENKIEwtc[, The Finite Element Method in Engineering Science. McGraw-Hill, London (1971).

7. S. Trsosi*LNKo and J. N. GOODEER, Theory of Elasticity, 2nd Edn. McGraw-Hill, New York (1951). 